\title{
Consequences of a Generalized Newtonian Gravity with an Exponential Factor
}

\author{
Lenser Aghalovyan \\ Institute of Mechanics of National Academy of Sciences of Armenia, Yerevan, Armenia \\ Email: lagal@sci.am
}

How to cite this paper: Aghalovyan, L. (2020) Consequences of a Generalized Newtonian Gravity with an Exponential Factor. International Journal of Astronomy and Astrophysics, 10, 224-234.

https://doi.org/10.4236/ijaa.2020.103012

Received: May 27, 2020

Accepted: September 5, 2020

Published: September 8, 2020

Copyright $\odot 2020$ by author(s) and Scientific Research Publishing Inc. This work is licensed under the Creative Commons Attribution International License (CC BY 4.0).

http://creativecommons.org/licenses/by/4.0/

\begin{abstract}
The central interaction of bodies is investigated, which enhances the Newtonian interaction by the exponential factor. As a consequence, it has been shown that Black Holes are subordinate to this enhanced interaction. All Black Holes can be systematized in accordance with their mass, the radius of the event horizon and the gravitational field intensity exponent, created by the Black Hole.
\end{abstract}

\section{Keywords}

Gravitation, Central Interaction, Potential Field, Escape Velocity, Black Hole, Density

\section{Introduction}

In order to build an acceptable theory of the solar system planetary motion, humanity has passed a long, centuries-old path. In the III century B.C. the representative of Pythagorean school of Greece Aristarcus of Samos (Aristarcus of Samos, 310-230 B.C.) put forward the heliocentric system of the planetary motion, but it was rejected by the ancient astronomers, as in their opinion it was baseless. Later on, the most famous one was the geocentric system of Ptolomey (Claudius Ptolomey, 90-168 A.D.), who lived in Alexandria in the II century A.D. From the observations of the starry sky, the ancients concluded that it goes around our Earth, which was considered to be motionless and in the center of the Universe. In the system of Ptolemey everything is explained with the help of circles and circular motions. But this system, which existed over a thousand years, turned out to be very complicated and regularly came into conflict with the data of the astronomic observations, which became intensive after the invention of the telescope by Galileo Galilei (Galileo Galilei, 1564-1642). 
In the XVI century Copernicus (Nicolaus Copernicus, 1473-1543) revived the heliocentric model and unlike Aristarchus, who only expressed the general idea, he developed the details of the heliocentric model and the basics of the planetary positions calculations. Yet, Copernicus continued to rely on the method of Ptolomey's circular orbit, using 48 circles instead of 40 as Ptolomey did. Copernicus' theory allowed the interpretations of the observations to be more exact in some cases, and less in others. Copernicus' heliocentric model was as bulky and complex as competing with it the geocentric model and didn't differ by great accuracy. The radical change in the victory of the heliocentric system introduced Johann Kepler (Johann Kepler, 1571-1630) at the beginning of the XVII century. Based on the wonderful catalog, composed by Tycho Brahe (Tycho Brahe, 1546-1601) with the data of the exclusively exact observations connected with the planetary motion, in particular, data for Mars, at the beginning Kepler formulated the first two of his three famous laws and some years later as well the third law. According to Kepler first law any planet of the solar system moves around the Sun in an elliptical orbit with the Sun in one of the focuses of the ellipse. With this Ptolomean geocentrical model of motion was disproved. According to the second law any planet moves in the orbit with constant sectorial speed, i.e. the straight line, connecting the planet with the Sun, outlines equal areas during equal time interval. The third law establishes the connection between the big semi-axis (a) of the ellipse and the period $(T)$, during which the planet completes a full turn: $\frac{T^{2}}{a^{3}}=\frac{4 \pi^{2}}{G(M+m)}, G$, gravitational constant. Kepler's laws are taken as empirical. Several decades later Newton (Isaac Newton, 1642-1717) mathematically derived Kepler's laws and formulated the famous gravity law. According to this law the force of the gravity is central and each mass $m$ is gravitated by another mass $M$ in the Universe with force inversely proportional to the square of the distance between the masses and is directed along the line, connecting the centers of the masses. Newton's another important achievement was that he proved that the orbit of the bodies moving around the Sun, may be any of the curves of the conical sections family (circle, ellipse, parabola, hyperbola). In the next decades and centuries Newton's gravity law has received a lot of convincing and vivid confirmations. We note some of them. Edmond Halley (Edmond Halley, 1656-1742), based on Newton's gravity law, predicted the next appearance in the sky of the Earth (December, 1758) the comet Halley (called by his name in 1759, it appears in the Sky of the Earth with the period of 75 - 76 years, the coming appearance is expected in 2061), observed since the ancient times (in 240 B.C.). Halley kept friendly contacts with Newton and at his own expenses in 1687 published Newton's famous work "Mathematical beginnings of natural philosophy", where Newton's gravity law was first stated. It is interesting that Halley did not live up to the date of his prediction. Yet his prediction became the first successful confirmation of Newton's Celestial Mechanics and clear demonstration of its predictive power. Leaning on New- 
ton's law William Herschel (William Herschel, 1738-1822) opened the planet Uranus (1781) and two satellites of Saturn (1789), measured the rotation period of Saturn and its rings (1790). English mathematician and astronomer Adams (John Adams, 1819-1892) and French astronomer and mathematician Le Verrier (Jean Le Verrier, 1811-1877) having studied the irregularities in the motion of the planet Uranus, came to a conclusion that it is the result of the influence of an unknown yet planet. They, independently from each other calculated it position and in 1846 discovered the planet Neptune. German astronomer Galle (Johann Galle, 1812-1910) discovered the planet in the sky in the place, indicated by Le Verrier. In the XVIII century lots of small planets, asteroids and satellites of the well-known planets were discovered.

In spite of many successes, some phenomena were difficult to explain by Newton's law. In 1859 Le Verrier detected some discrepancy in the orbit of the planet, closest to the Sun, Mercury at perigee with the observations results. Well-known American astronomer Simon Newcomb (Simon Newcomb, 1835-1909) not finding convincing explanations of this fact, in 1895 expressed an opinion, that it is possible Newton's inverse squares law is not fulfilled exactly at small distances. After having built the General theory of relativity (GRT) by Einstein (Albert Einstein, 1879-1955) in 1917 some explanation was given to the problem with Mercury and it seemed that the problem was solved. But in 1965 Dikke R. and Goldenberg M. proved that the Sun is not spherical and its polar diameter is 35 $\mathrm{km}$ less than the equatorial. It permitted to explain the part of the residual perihelion displacement of Mercury, which put under suspicion the agreement of GRT with the results of the observations.

There is also the second peculiarity of the current situation, which is connected with the question of the existence of the Black Hole ("Dark Body"). English astronomer amateur, one of the founders of seismology John Mitchell (John Mitchell, 1724-1793) in 1783 and well-known French mathematician and mechanic Laplass (Pierre Laplass, 1749-1827) in 1795, on the base of Newton's theory of gravity, independently from each other expressed an opinion that in the nature should exist bodies and to overcome their gravity the necessary velocity should exceed the velocity of light $(c)$. Therefore such bodies should be "dark", i.e. invisible. They may be discovered in an indirect way by the gravitation influence on other bodies. Mitchell and Laplass derived radius of the "Dark Body" $r_{g}=2 G M / c^{2}$ (gravitational radius) for its given mass, applying the concept of the second cosmic velocity (escape velocity). GRT supporters criticized Mitchell and Laplass considerations, in the sence, that at the velocities close to the velocity of light the formulae of classical mechanics are not applicable, though by both theories one and the same value of the gravitational radius $r_{g}$ is obtained. And the opponents of GRT affirm that this theory is not applicable, as the solution of its equations has singularity, unacceptable when describing natural phenomena.

In this connection, we give an opinion of Peter Bergman, Albert Einstein's 
student and fellow campaigner:

"In the solar system and even in our entire Galaxy, the relative motions of the bodies entering these formations are so slow that it is practically indifferent whether to choose the Galaxy as the Lorentz's system or the system in which is resting the center of inertia of the solar's system. The neglected smallness of relativistic effects may explain why the use of Newtonian methods of calculation leads to extremely satisfacting results" [1].

Unfortunately an acceptable clarity has not been contributed on these problems until recent times [1] [2] [3] [4].

In the papers [5] [6] a new type of central interaction is determined, generalizing Newtonian interaction of gravity. This interaction at short distances is stronger than Newtonian interaction and practically coincides with it at long distances. This confirms the assumption of the famous astronomer Newcomb. In the given paper some important properties of the potential field, created by generalized-Newtonian gravity force [6] are described, the problem of Black Holes (“Dark Bodies") is interpreted in a new way.

\section{Generalized Newtonian Interaction of World-Wide Gravitation}

Let us have bodies with mass $M, m$. We place the beginning of the polar coordinates $(r, \theta)$ in the center of the body with mass $M$. We set the central force of interaction between these bodies in the form [5] [6]

$$
F=-G m M \frac{\mathrm{e}^{k / r}}{r^{2}} \frac{r}{|r|}
$$

or

$$
F=-G m M \frac{\mathrm{e}^{k / r}}{r^{2}}
$$

where $G$ is the gravitational constant in Newton gravity law $\left(G=6.67 \times 10^{-11} \mathrm{~m}^{3} /\left(\mathrm{kg} \cdot \mathrm{s}^{2}\right)\right)$. Index $k$ characterizes the power (intensity) of the gravity center and has length dimension. At $k=0$ interaction (1), (2) coincides with Newtonian interaction $\left(F=-(G m M) / r^{2}\right)$, taking this into account, in (1) coefficient $G m M$ is introduced. At $k>0$ interaction (1) for short distances is more powerful than Newtonian interaction. At long distances the both interactions practically coincide. Since $F$ is central force, material point trajectory of the mass " $m$ " is plane curve and law of areas takes place. Created by force $F$ the field is potential with potential

$$
U=-\frac{G m M}{k} \mathrm{e}^{k / r}+\text { const }
$$

which is essentially stronger than the potential of Newtonian field $(U=-G m M / r)$.

Using the theorem on kinetic energy $\left(\frac{\mathrm{d} m v^{2}}{2}=F \mathrm{~d} r\right)$, according to (2) we 
have

$$
v^{2}=\frac{2 G M}{k} \mathrm{e}^{k / r}+h
$$

where the constant of integration $h$ is determined from the initial condition: at $r=r_{0}, v=v_{0}$

$$
h=v_{0}^{2}-\frac{2 G M}{k} \mathrm{e}^{k / r_{0}}
$$

Trajectory of the body motion with mass $m$ is conic section [6]

$$
r=\frac{1 / k_{1}}{1-\frac{k_{2}}{k_{1}} \cos \sqrt{\delta_{1}}\left(\theta-\theta_{0}\right)}
$$

where

$$
\begin{gathered}
k_{1}=\frac{G M}{C^{2} \delta_{1}}, \delta_{1}=1-\frac{G M k}{C^{2}}>0 \\
k_{2}^{2}=\frac{1}{k C^{2} \delta_{1}^{2}}\left[G M+(G M+k h) \delta_{1}\right]
\end{gathered}
$$

constant $C$ is equal to the moment value of the initial velocity, relative to the center of gravity. The parameters of the conical section are determined by formulas:

$$
\begin{aligned}
& p=\frac{1}{k_{1}}=\frac{C^{2}}{G M}-k \\
& \varepsilon=\frac{k_{2}}{k_{1}}=\sqrt{1+\left(\frac{C^{2}}{M G k}-1\right)\left(2+\frac{h k}{M G}\right)}
\end{aligned}
$$

the movement trajectory will be an ellipse, if

$$
-\frac{M G}{k}\left(1+\frac{C^{2}}{C^{2}-M G k}\right)<h<-\frac{2 G M}{k}
$$

the semi-axis of the ellipse are determined by formulas

$$
a=\frac{p}{1-\varepsilon^{2}}, b=\frac{p}{\sqrt{1-\varepsilon^{2}}}
$$

The trajectory is parabola at $h=-\frac{2 G M}{k}$ and hyperbola at $h>-\frac{2 G M}{k}$.

Applying formula (5) the condition $h<-\frac{2 G M}{k}$ for the elliptic trajectory is written in the form of

$$
\begin{gathered}
v_{0}^{2}<v_{*}^{2}, v_{*}^{2}=\frac{2 G M}{r_{0}}\left(\frac{\mathrm{e}^{k / r_{0}}-1}{k / r_{0}}\right) \\
\lim _{k \rightarrow 0} v_{*}^{2}=\frac{2 G M}{r_{0}}=V_{N}^{2}, V_{N}=\sqrt{\frac{2 G M}{r_{0}}} \text { is the second cosmic velocity (escape }
\end{gathered}
$$

velocity) by Newton theory, i.e. the initial velocity at which the body of mass $m$ 
overcomes the gravity of the body of mass $M$. If $k>0$ from (11) follows $V_{*}>V_{N}$, i.e. with interaction (1) the escape velocity is more than the classical one. Summarizing, we can state that there is a central interaction of bodies, such as (1), which at short distances is more powerful than the Newtonian interaction. This confirms prediction of Newcomb, made on the problem of perihelium of planet Mercury. In the framework of interaction (1), this problem requires a separate detailed consideration, in accordance with the approaches outlined in the Roseveare's monograph [7].

We determine the gravitational radius $\left(R_{g}\right)$, which corresponds to the interaction (1). The body with mass $M$ will be "Black Hole" ("Dark", invisible), if any body with mass $m$ and initial velocity, even equal to velocity of light " $c$ " cannot overcome the field of gravity of the mass $M$. The initial conditions of the problem will be: at $r_{0}=R_{g}, v_{*}=c$. Substituting these conditions into (11), denoting $\lim _{k \rightarrow 0} R_{g}=r_{g}$ we shall have

$$
R_{g}=r_{g} \frac{\mathrm{e}^{\gamma}-1}{\gamma} \text { or } \frac{R_{g}}{r_{g}}=\frac{\mathrm{e}^{\gamma}-1}{\gamma}
$$

where

$$
r_{g}=\frac{2 G M}{c^{2}}, \gamma=\frac{k}{R_{g}}
$$

i.e. $r_{g}$ is the well-known gravitational radius at Newtonian classical central interaction. It is obvious that at $\gamma \neq 0, R_{g}>r_{g}$ and according to (12) depending on $\gamma, R_{g}$, may be arbitrarily big, comparing with $r_{g}$ (see graph of function $R_{g} / r_{g}$, (table 1, table 2) [6]). For the "Black Hole" the interaction (1) according to (13) has the form of

$$
F=-G m M \frac{\mathrm{e}^{\gamma \frac{R_{g}}{r}}}{r^{2}} \frac{r}{|r|}
$$

"Black Holes" (in our opinion term "Dark Bodies" is more suitable) will differ from each other by mass, gravitational radius $R_{g}$ and gravitational field intensity exponent $\gamma$. According to graph of function $R_{g} / r_{g}$ (see Figure 1), arbitrarily many "Black Holes" may exist. The astronomers and astrophysics proved this [8]-[13], as in any galaxy there is a center (supposedly "Black Hole"), round which as well turn stars [14] [15] [16]. The mass of such a body comparing with the Sun mass $(\odot), \odot \approx 2 \times 10^{30} \mathrm{~kg}$, is very big. For example, for the Black Hole S50014+81 $M=4 \times 10^{10} \odot$ [17], for NGC $6166 M=3 \times 10^{10} \odot$ [18], for NGC $1277 M=1.2 \times 10^{9} \odot$ [19], for Swan A $M=1 \times 10^{9} \odot$ [20]. The existence of such centers (formations) create harmony in the Universe, like harmony in the Sun system, but in huge measures. They, particularly, in every way prevent "the escape" of the stars and their systems, with also preventing the Universe expansion, as well. The generalized-Newtonian theory of gravity turned out to be capable to clarify this complicated problem, with this again denoting Newton's greatness. 


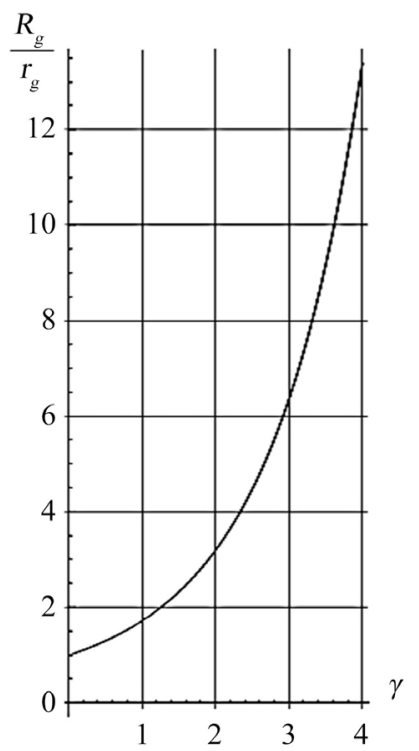

Figure 1. The dependence between $R_{g} / r_{g}$ and the gravitational field intensity exponent $\gamma$.

\section{On Horizon of Events}

The horizon of events is called the boundary (surface) of the space area, the gravity of which is so great, that even the objects, moving with velocity of light (c) cannot leave it. Usually this area is considered to be sphere, the radius of which coincides with the gravitational radius. The radius determined from the relation of the area surface $S$ of this sphere to $(4 \pi)$, i.e. $R_{g}^{2}=S / 4 \pi$, is called gravitational radius of Schwarzschild (Karl Schwarzschild, 1873-1916), who in 1916 introduced the concept "horizon of events". By Hawking (Stephen Hawking, 1942-2018) the horizon of events is made of light, which is not able to leave the Black Hole and that is why "soars" on this horizon [21]. By Newton theory and by GRT gravitational radius $r_{g}=2 G M / c^{2}$. The Earth can become a Black Hole if in some way its whole mass is succeeded to be put into a ball with radius of 9 $\mathrm{mm}$, for the Sun $3 \mathrm{~km}$, which is difficult to imagine. In the presence of interaction (1) the gravitational radius $R_{g}$ is determined by formula (12) and its value depends as on the mass, as well as on the gravitational field intensity exponent $\gamma$. Above we showed that $R_{g}$ comparing with Newtonian $r_{g}$ can be arbitrarily large. I.e. by interaction (14) the Black Hole will have real dimensions, which is principally observed in reality for massive Black Holes [8]-[20]. Taking as basis of the calculations the gravitational radius of Schwarzschild $\left(R_{g}^{2}=S / 4 \pi\right)$ and predicting by observations mass $M$ of the assumed Black Hole (some data are brought above) first determine $r_{g}=2 G M / c^{2}$, then $R_{g} / r_{g}$. Later, from the correlation (12) the value of gravitational field (14) intensity exponent $\gamma$ created by the Black Hole is determined. As an illustration, we present some values for the gravitational field intensity exponent $\gamma$ (Table 1), calculated from the relation (12). 
Table 1. The values of $\gamma$ corresponding to the given values of $R_{g} / r_{g}$.

\begin{tabular}{cccccc}
\hline$R_{g} / r_{g}$ & 1 & 2 & 3 & 5 & 10 \\
\hline$r$ & 0 & 1.256 & 1.904 & 2.66 & 3.615 \\
& 50 & 100 & 200 & 500 & 1000 \\
& 5.647 & 6.475 & 7.285 & 8.335 & 9.118 \\
\hline
\end{tabular}

So, interaction (14) permits to systematize all the well-known (presupposed) Black Holes (see the corresponding catalog of Black Holes) as by the size of their mass, as well as by the radius of the horizon of events $R_{g}$ (Schwarzchild's gravitational radius) and the gravitational field intensity exponent $\gamma$. We also note that since, according to Newton's theory and GRT, the main characteristic of the field is $r_{g}=2 G M / c^{2}$, it is impossible to systematize existing Black Holes by these theories; moreover, Black Holes create a stronger force field (14) near them.

\section{On Density of Black Hole}

By Newtonian theory of gravity the medium density of the Black Hole $\rho_{N}$ is calculated by formula $\rho_{N}=M / V, V=\frac{4}{3} \pi r_{g}^{3}$, where $V$ is the volume of the ball, corresponding to the horizon of events, i.e.

$$
\rho_{N}=\frac{3 c^{6}}{32 \pi G^{3} M^{2}}
$$

In case of generalized-Newtonian interaction (14) the medium density $\rho$ of the Black Hole is calculated by formula

$$
\rho=\frac{3 M}{4 \pi R_{g}^{3}}=\frac{\rho_{N}}{\lambda_{\gamma}^{3}}, \lambda_{\gamma}=\frac{\mathrm{e}^{\gamma}-1}{\gamma}
$$

at $\gamma>0, \lambda_{\gamma}>1$, the density of the Black Hole in $\lambda_{\gamma}^{3}$ times will be less than the density $\rho_{N}$. At $\gamma \gg 1, \rho \ll \rho_{N}$. The volume of the Black Hole

$$
V_{\text {b.h. }}=\frac{4}{3} \pi R_{g}^{3}=\frac{4}{3} \pi r_{g}^{3} \lambda_{\gamma}^{3}
$$

is much more than the volume of the Black Hole by Newtonian theory.

The above results allow to conclude that the real Black Holes will have a density much lower than the density and the volume much larger than the volume according to Newton's theory.

\section{On Accelerations of Bodies in the Force Field of Gravity}

\section{(1)}

The force field created by Newtonian force of gravity $\left(F=-G m M / r^{2}\right)$ has a remarkable property, that all the bodies, being at the same distance from the center of gravity, independent from their dimensions and mass, get from field of the same acceleration [1]. We shall prove that the field, created by force interac- 
tion has this property (1), as well.

Let the body with the mass $M$ be the center of gravity by (1) and we have bodies with the masses $m_{1}, m_{2}$, being at the distance $r_{0}$ from the center of gravity. Then according to (2), at $r=r_{0}$ we have

$$
F_{1}=-\frac{G m_{1} M \mathrm{e}^{k / r_{0}}}{r_{0}^{2}}, F_{2}=-\frac{G m_{2} M \mathrm{e}^{k / r_{0}}}{r_{0}^{2}}
$$

Therefore

$$
\frac{F_{1}}{F_{2}}=\frac{m_{1}}{m_{2}}
$$

According to Newton second law of Mechanics

$$
m_{1} w_{1}=F_{1}, m_{2} w_{2}=F_{2}
$$

where $w_{1}, w_{2}$ are the accelerations of the bodies with masses $m_{1}, m_{2}$. From here it follows

$$
\frac{F_{1}}{F_{2}}=\frac{m_{1}}{m_{2}} \frac{w_{1}}{w_{2}}
$$

Matching (21) with (19) we have, $w_{1} / w_{2}=1$ i.e. two bodies, having arbitrary masses $m_{1}, m_{2}$ and dimensions, being at the same distance from the center of gravity, under the action of the field get the same accelerations.

\section{On Gravity Force}

Let us imagine that the body of mass $m$ is located on the surface of the Black Hole. Let's find out what its weight will be. The force of gravity (14) of the Black Hole acts on this body. Having accepted $r=R_{g}$, we will have

$$
F=-\frac{G m M}{R_{g}^{2}} \mathrm{e}^{\gamma}
$$

On the other hand $F=-m g$, where $g$ - gravity acceleration. Consequently we will have

$$
g=g_{N} \mathrm{e}^{\gamma}, g_{N}=\frac{G M}{R_{g}^{2}}
$$

where $g_{N}$ acceleration of gravity according to Newton's theory. From (23) it follows that the weight of the body $(P=m g)$, located on the surface of the Black Hole, can be arbitrarily big, depending on the value of the field intensity exponent $\gamma$, created by the Black Hole.

\section{Discussion and Conclusions}

A new type of central interaction of bodies has been established, generalizing the Newtonian interaction of world-wide gravitation. The prediction of famous astronomer Newcomb is verified. It is proved that with such an interaction, the trajectory of the body is a conical section, however, the escape speed is much more than the speed at Newtonian interaction. The close relationship of this in- 
teraction with the Black Holes problem is shown.

The gravitational field created by the Black Hole obeys the generalized-Newtonian interaction: $F=-G m M \frac{\mathrm{e}^{\frac{\gamma_{g}}{r}}}{r^{2}} \frac{r}{|r|}$. Could exist any number of Black Holes, which will differ by mass, radius of the event horizon (Schwarzchild's gravitational radius) and gravitational field intensity exponent $\gamma$ ? The Schwarzchild's gravitational radius $R_{g}$ can be arbitrarily larger than the Newtonian's gravitational radius $r_{g}$. At generalized-Newtonian interaction (14), the density of Black Holes is significantly lower than the density at Newtonian interaction. All Black Holes can be systematized according to their mass $M$, Schwarzchild's gravitational radius $R_{g}$, gravitational field intensity exponent $\gamma$.

The solution corresponding to the Generalized-Newtonian Force of World-Wide Gravitation does not contain the singularity.

Newton's theory of gravity also sheds light on the problem of Black Holes. For this should use the generalized-Newtonian interaction (14).

\section{Conflicts of Interest}

The author declares no conflicts of interest regarding the publication of this paper.

\section{References}

[1] Bergman, P.G. (1968) The Riddle of Gravitation. Charles Scribner's Sons, New York.

[2] Layzer, D. (1984) Constructing the Universe. Scientific American Books Inc., New York.

[3] Kittel, C., Knight, W.D., et al. (1965) Mechanics (Berkley Physics Course, Vol. 1). McGraw-Hill, New York.

[4] Wichmann, E. (2010) Quantum Physics (Berkeley Physics Course, Vol. 4). McGraw-Hill, India.

[5] Aghalovyan, L.A. (2006) On Universal Central Strong (Weak) Interaction of Bodies and Particles. Reports of National Academy of Sciences of Armenia, 106, 238-244.

[6] Aghalovyan, L.A. (2018) The Consequences of a Strengthened Newtonian Gravity at Short Distances. International Journal of Astronomy and Astrophysics, 8, 191-199. https://doi.org/10.4236/ijaa.2018.82014

[7] Roseveare, N.T. (1982) Mercury's Perihelion from Le Verrier to Einstein. Clarendon Press, Oxford.

[8] McConnell, N., Ma, C., et al. (2011) Two Ten-Billion-Solar-Mass Black Holes at the Center of Giant Elliptical Galaxies. Nature, 480, 215-218. https://doi.org/10.1038/nature10636

[9] Ellis, A. (1999) Black Holes-Part 1-History. The Astronomical Society of Edinburgh Journal, 39, 9.

[10] Helh, F.W., Kiefer, C., et al. (1998) Black Holes: Theory and Observation. In: Hehl, Friedrich, W., Kiefer, Claus, Metzler and Ralph, J.K., Eds., Proceedings of the 179 th 
W.E. Heraeus Seminar Held at Bad Honnef, Germany, 18-22 August 1997.

[11] Serabyn, E. and Lacy, J. (1985) NE II Observations of the Galactic Center: Evidence for a Massive Black Hole. The Astronomical Journal, 293, 445-458. https://doi.org/10.1086/163250

[12] Ferrarese, L. and Merritt, D. (2002) Supermassive Black Holes. Physics World, 15, 4-46. https://doi.org/10.1088/2058-7058/15/6/43

[13] Mitra, A. (2012) Kruskal Coordinates and Mass of Schwarzschild Black Holes: No Finite Mass Black Holes at All. International Journal of Astronomy and Astrophysics, 2, 236-248. https://doi.org/10.4236/ijaa.2012.24031

[14] Eckart, A. and Genzel, R. (1996) Observations of Stellar Proper Motions near the Galactic Centre. Nature, 383, 415-417. https://doi.org/10.1038/383415a0

[15] Gillessen, S., Eisenhauer, F., et al. (2009) Monitoring Stellar Orbits around the Massive Black Hole in the Galactic Centre. The Astrophysical Journal, 692, 1075-1109. https://doi.org/10.1088/0004-637X/692/2/1075

[16] Genzel, R., Schödel, R., et al. (2003) The Stellar Cusp around the Supermassive Black Hole in the Galactic Centre. The Astrophysical Journal, 594, 812-832. https://doi.org/10.1086/377127

[17] Ghisellini, G., Ceca, R., et al. (2010) Chasing the Heaviest Black Holes in Active Galactic Nuclei, the Largest Black Hole. Monthly Notices of the Royal Astronomical Society, 405, 387-400. https://doi.org/10.1111/j.1365-2966.2010.16449.x

[18] Magorrian, J., Tremaine, S., et al. (1998) The Demography of Massive Dark Objects in Galaxy Centers. The Astronomical Journal, 115, 2285-2305. https://doi.org/10.1086/300353

[19] Graham, A.W., Durre M., et al. (2016) A Normal Supermassive Black Hole in NGC 1277. The Astrophysical Journal, 819, 43. https://doi.org/10.3847/0004-637X/819/1/43

[20] (2015) Black Holes: Gravity's Relentless Pull Interactive: Encyclopedia-Hubble Site.

[21] Hawking, S. (1996) Black Holes and Baby Universes and Other Essays. Bantam Book, Toronto. 